\title{
Natural frequencies for bending vibrations of Timoshenko cracked beams
}

\author{
J.A. Loya ${ }^{\mathrm{a}}$, L. Rubio ${ }^{\mathrm{b}}$, J. Fernández-Sáez,* \\ ${ }^{a}$ Department of Continuum Mechanics and Structural Analysis, Carlos III University of Madrid, Avda. de la Universidad, \\ 30. 28911 Leganés, Madrid, Spain \\ ${ }^{\mathrm{b}}$ Department of Mechanical Engineering, Carlos III University of Madrid, Avda. de la Universidad, \\ 30. 28911 Leganés, Madrid, Spain
}

\begin{abstract}
The natural frequencies for bending vibrations of Timoshenko cracked beams with simple boundary conditions have been obtained. The beam is modelled as two segments connected by two massless springs (one extensional and another one rotational). This model promotes discontinuities in both vertical displacement and rotation due to bending, which are proportional to shear force and bending moment transmitted by the cracked section, respectively. The differential equations for the free bending vibrations are established and then solved individually for each segment with the corresponding boundary conditions and the appropriated compatibility conditions at the cracked section. The problem is also solved by the perturbation method and both procedures are applied to the case of a simply supported cracked beam. The results show that the perturbation method provides simple expressions for the natural frequencies of cracked beams and it gives good results for shallow cracks.
\end{abstract}

\footnotetext{
*Corresponding author Tel.: + 3416249964 ; fax: + 3416249430 .

E mail address: ppfer@ing.uc3m.es (J. Fernández Sáez).
} 


\section{Introduction}

It is well known that cracks reduce the natural frequencies of a structure because it becomes more flexible. This fact has been extensively used to detect the presence, size and location of cracks in a structural component. (See, for instance Refs. [1 14].)

The analysis to detect, locate and quantify the extent of damages in beams from the knowledge of their vibratory characteristics falls within what is known as "inverse problems". To address the solutions of an inverse problem it is necessary to know the solutions of the named "direct problem", i.e. the determination of natural frequencies of cracked beams knowing the crack parameters.

Natural frequencies of cracked beams can be obtained by 2-D or 3-D numerical analysis using the Finite Element Method (FEM). Alternatively, simplified procedures are available with less computational effort. Among these simplified methods are those proposed by Christides and Barr [15] and Shen and Pierre [16,17]. In both cases, a crack function representing the perturbation in the stress field induced by the crack is considered. Chondros et al. [18] have developed a continuous cracked beam vibration theory. They considered that crack introduces a continuous change in the flexibility in its neighborhood and models it by incorporating a displacement field consistent with the singularity.

In other cases, the cracked beam is modelled as two segments connected by means of massless rotational springs [19], whose stiffness may be related to the crack length by the Fracture Mechanics theory [20]. Thus, at the cracked section a discontinuity in the rotation due to bending must be considered. This kind of models has been successfully applied to Euler Bernoulli cracked beams with different support conditions [6,21 25].

The perturbation method has been used by other authors $[3,26,27]$ to calculate the variation of natural frequencies induced by cracks in Euler Bernoulli beams.

For the case of Timoshenko beams, where the effects of shear deformation and rotary inertia are non-negligible, Carneiro and Inman [28] have developed a continuous model for the transverse vibrations of the cracked beam.

Lele and Maiti [10] have modelled the presence of a crack by means a rotational spring as in the Euler Bernoulli cracked beam problem. Krawczuk et al. [29] used the same crack model to develop a spectral finite element for the Timoshenko cracked beam. However, the cited authors do not consider any discontinuity in the transverse deflection at the cracked section. Nevertheless, this discontinuity must be taken into account from the analysis of local flexibility of a cracked beam element (according to Okamura et al. [30] and Tharp [31]). On the other hand, to the author's knowledge the perturbation method has not been used to obtain the natural frequencies of bending vibrations of Timoshenko cracked beams.

In this paper, natural frequencies of bending vibrations of Timoshenko cracked beams have been obtained. The beam is modelled as two segments connected by two massless springs (one extensional and another one rotational). The differential equations for the free bending vibrations are established and then solved individually for each segment with the corresponding boundary conditions and the proper compatibility conditions at the cracked sections. The compatibility conditions include a discontinuity in the rotation due to bending as well as a discontinuity of the transverse deflection at the cracked section. This problem has also been solved by the perturbation theory.

Finally, both methods are used to obtain solutions for the case of a simply supported cracked beam. 


\section{Problem formulation}

Consider an uncracked Timoshenko beam of length $L$, uniform cross-section $A$ and moment of inertia about the neutral axis, $I$, with specified boundary conditions. The coupled equations of the free bending vibrations of this beam are given by [32]:

$$
\begin{gathered}
\rho A \frac{\partial^{2} v(x, t)}{\partial t^{2}}=\kappa G A\left(\frac{\partial^{2} v(x, t)}{\partial x^{2}}-\frac{\partial \psi(x, t)}{\partial x}\right), \\
\rho I \frac{\partial^{2} \psi(x, t)}{\partial t^{2}}=\kappa G A\left(\frac{\partial v(x, t)}{\partial x}-\psi(x, t)\right)+E I \frac{\partial^{2} \psi(x, t)}{\partial x^{2}},
\end{gathered}
$$

where $v(x, t)$ and $\psi(x, t)$ represent, respectively, the transverse deflection of the beam and the rotation due to bending, $\rho$ is the material mass density, $E$ is the Young modulus, $G$ is the shear modulus and $\kappa$ is a coefficient introduced to account for the geometry-dependent distribution of shear stress.

The solution can be found by using the separation of variables method as:

$$
v(x, t)=L V(x) \mathrm{e}^{\mathrm{i} \omega t}, \psi(x, t)=\Psi(x) \mathrm{e}^{\mathrm{i} \omega t},
$$

where $\omega$ is the natural frequency of vibrations. Substituting Eq. (3) into Eqs. (1) and (2) and using the new variables and constant given by

$$
\xi=\frac{x}{L}, \quad \lambda^{2}=\frac{\rho A}{E I} L^{4} \omega^{2}, \quad r^{2}=\frac{I}{A L^{2}}, \quad s^{2}=\frac{E I}{\kappa A G L^{2}},
$$

Eqs. (1) and (2) are transformed into

$$
\begin{gathered}
V^{\prime \prime}(\xi)+\lambda^{2} s^{2} V(\xi)-\Psi^{\prime}(\xi)=0, \\
s^{2} \Psi^{\prime \prime}(x)-\left(1-\lambda^{2} r^{2} s^{2} \Psi(\xi)\right)+V^{\prime}(\xi)=0,
\end{gathered}
$$

where $(\cdot)^{\prime}$ represents the derivative with respect to $\xi$. These equations must be solved with the boundary conditions depending on the end supports as follows:

Simply supported end

$$
V=\Psi^{\prime}=0
$$

Fixed end

$$
V=\Psi=0 .
$$

Free end

$$
\Psi^{\prime}=0, \quad V^{\prime}-\Psi=0 .
$$

The solution of this problem can be found, for instance, in Ref. [33].

Consider now that the beam has a crack of length $a$ located at a distance $L^{*}\left(e=L^{*} / L\right)$ from the left support. It is assumed that the crack always remains open. The cracked beam has been treated as two beams connected by two elastic springs (a rotational and an extensional) at the cracked section (Fig. 1). The stiffness of the springs depends on the crack depth and on the geometry of the cracked section. This model leads to discontinuities in both vertical displacement 
and rotation due to bending, proportional to shear force and bending moment transmitted by the cracked section, respectively, i.e. [30,31]

$$
\begin{gathered}
\Delta v=\left.C_{q} \kappa G A\left(\frac{\partial v(x, t)}{\partial x}-\psi(x, t)\right)\right|_{x=L^{*}}, \\
\Delta \psi=\left.C_{m} E I \frac{\partial \psi(x, t)}{\partial x}\right|_{x=L^{*}} .
\end{gathered}
$$

$C_{q}$ and $C_{m}$ are the flexibility constants of the springs and can be calculated as

$$
\begin{aligned}
C_{q} & =\frac{W}{E A} q(\alpha, \text { geometry of the cross-section }), \\
C_{m} & =\frac{W}{E I} \Theta(\alpha, \text { geometry of the cross-section }),
\end{aligned}
$$

where $W$ is the width of the beam, $q(\alpha)$ and $\Theta(\alpha)$ are functions depending on the crack ratio $\alpha=a / W$ and the beam cross-section geometry. In the case of a rectangular section, the function $q(\alpha)$ can be written as [34]

$$
q(\alpha)=\left(\frac{\alpha}{1-\alpha}\right)^{2}\left(-0.22+3.82 \alpha+1.54 \alpha^{2}-14.64 \alpha^{3}+9.60 \alpha^{4}\right)
$$

and the $\Theta(\alpha)$ function has the form [35]

$$
\Theta(\alpha)=2\left(\frac{\alpha}{1-\alpha}\right)^{2}\left(5.93-19.69 \alpha+37.14 \alpha^{2}-35.84 \alpha^{3}+13.12 \alpha^{4}\right) .
$$

In this way, the strain energy of the cracked beam should be the same as that of the two beams connected by the two springs. The contribution of the extensional spring to the strain energy of the system is small in comparison with that of the rotational spring and many authors neglect this effect to analyze the cracked beam dynamic behavior $[10,14,29]$. However, we have included here the presence of an extensional spring at the crack location (and the corresponding discontinuity in the vertical displacement) for coherency with the general derivation of compliance for the cracked beams presented by Okamura et al. [30], (see also the work by Tharp [31]).

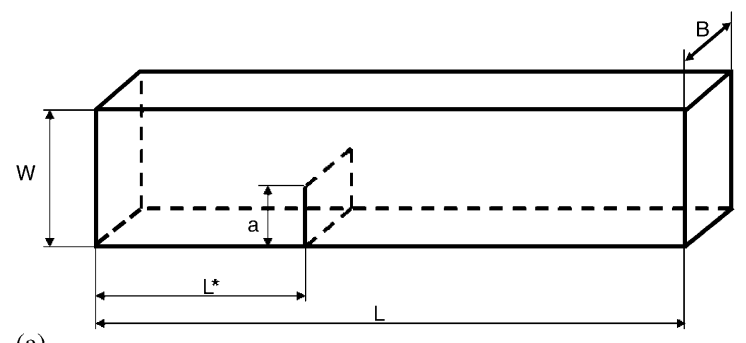

(a)

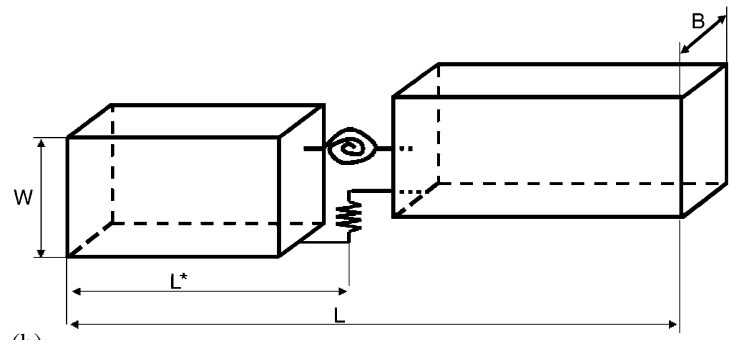

(b)

Fig. 1. Beam with a transverse edge crack: (a) Dimensions of the beam and position of the crack, (b) model for the cracked beam. 
Thus, the equations for the two segments are the following:

$$
\begin{gathered}
V_{1}^{\prime \prime}(\xi)+\Lambda^{2} s^{2} V_{1}(\xi)-\Psi_{1}^{\prime}(\xi)=0, \quad 0<\xi<e, \\
s^{2} \Psi_{1}^{\prime \prime}(x)-\left(1-\Lambda^{2} r^{2} s^{2} \Psi_{1}(\xi)\right)+V_{1}^{\prime}(\xi)=0, \quad 0<\xi<e, \\
V_{2}^{\prime \prime}(\xi)+\Lambda^{2} s^{2} V_{2}(\xi)-\Psi_{2}^{\prime}(\xi)=0, \quad e<\xi<1, \\
s^{2} \Psi_{2}^{\prime \prime}(x)-\left(1-\Lambda^{2} r^{2} s^{2} \Psi_{2}(\xi)\right)+V_{2}^{\prime}(\xi)=0, \quad e<\xi<1,
\end{gathered}
$$

$\Lambda$ being the frequency parameter of the cracked beam, that can be related to its natural frequency, $\omega_{c}$, by

$$
\Lambda^{2}=\frac{\rho A}{E I} L^{4} \omega_{c}^{2} .
$$

The above four equations must be solved with the specified boundary conditions and the following compatibility conditions at the cracked section:

Jump in the vertical displacement:

$$
\Delta V=V_{2}(e)-V_{1}(e)=\frac{W}{L} \frac{r^{2}}{s^{2}} q(\alpha)\left(V_{2}^{\prime}(e)-\Psi_{2}(e)\right) .
$$

Jump in the rotation due to bending:

$$
\Delta \Psi=\Psi_{2}(e)-\Psi_{1}(e)=\frac{W}{L} \Theta(\alpha) \Psi_{2}^{\prime}(e) .
$$

Continuity of the bending moment:

$$
\Psi_{2}^{\prime}(e)=\Psi_{1}^{\prime}(e)
$$

Continuity of the shear force:

$$
V_{2}^{\prime}(e)-\Psi_{2}(e)=V_{1}^{\prime}(e)-\Psi_{1}(e) .
$$

In the following sections, direct and approximate solutions obtained by the perturbation method are provided for the natural frequencies of the cracked beam.

\section{3. "Direct solution"}

A direct solution for the natural frequencies can be achieved by analyzing separately the two segments lying on either side of the crack. The solution for each segment $V_{1}(\xi)$ and $\Psi_{1}(\xi)$ for the left part and $V_{2}(\xi)$ and $\Psi_{2}(\xi)$ for the right part can be written as $[10,13,14]$

$$
\begin{gathered}
V_{1}(\xi)=C_{1} \cosh \beta_{1} \xi+C_{2} \sinh \beta_{1} \xi+C_{3} \cos \beta_{2} \xi+C_{4} \sin \beta_{2} \xi, \\
\Psi_{1}(\xi)=m_{1} C_{1} \sinh \beta_{1} \xi+m_{1} C_{2} \cosh \beta_{1} \xi-m_{2} C_{3} \sin \beta_{2} \xi+m_{2} C_{4} \cos \beta_{2} \xi, \\
V_{2}(\xi)=C_{5} \cosh \beta_{1} \xi+C_{6} \sinh \beta_{1} \xi+C_{7} \cos \beta_{2} \xi+C_{8} \sin \beta_{2} \xi, \\
\Psi_{2}(\xi)=m_{1} C_{5} \sinh \beta_{1} \xi+m_{1} C_{6} \cosh \beta_{1} \xi-m_{2} C_{7} \sin \beta_{2} \xi+m_{2} C_{8} \cos \beta_{2} \xi,
\end{gathered}
$$


$\beta_{1}, \beta_{2}, m_{1}$ and $m_{2}$ being

$$
\begin{aligned}
& \beta_{1}=\sqrt{-d_{1}+\sqrt{ }\left(d_{1}^{2}-d_{2}\right), \quad \beta_{2}=\sqrt{ } d_{1}+\sqrt{ }\left(d_{1}^{2}-d_{2}\right),} \\
& m_{1}=\frac{\beta_{1}^{2}+\Lambda s^{2}}{\beta_{1}}, \quad m_{2}=\frac{\beta_{2}^{2}-\Lambda s^{2}}{\beta_{2}},
\end{aligned}
$$

with

$$
d_{1}=\frac{\Lambda^{2}\left(r^{2}+s^{2}\right)}{2}, \quad d_{2}=\Lambda^{2}\left(r^{2} s^{2} \Lambda^{2}-1\right) .
$$

Expressions (22) (25) are valid for the case

$$
r^{2} s^{2} \Lambda^{2}<1
$$

If the above conditions are not satisfied, then

$$
\beta_{1}=\mathrm{j} / d_{1}-\sqrt{ }\left(d_{1}^{2}-d_{2}\right)=\mathrm{j} \beta_{1}^{\prime}
$$

with $\mathrm{j}=\sqrt{ }-1$, and the solutions are now given by

$$
\begin{gathered}
V_{1}(\xi)=C_{1} \cos \beta_{1}^{\prime} \xi+\mathrm{j} C_{2} \sin \beta_{1}^{\prime} \xi+C_{3} \cos \beta_{2} \xi+C_{4} \sin \beta_{2} \xi, \\
\Psi_{1}(\xi)=\mathrm{j} m_{1} C_{1} \sin \beta_{1}^{\prime} \xi+m_{1} C_{2} \cos \beta_{1}^{\prime} \xi-m_{2} C_{3} \sin \beta_{2} \xi+m_{2} C_{4} \cos \beta_{2} \xi \\
V_{2}(\xi)=C_{5} \cos \beta_{1}^{\prime} \xi+\mathrm{j} C_{6} \sin \beta_{1}^{\prime} \xi+C_{7} \cos \beta_{2} \xi+C_{8} \sin \beta_{2} \xi, \\
\Psi_{2}(\xi)=\mathrm{j} m_{1} C_{5} \sin \beta_{1}^{\prime} \xi+m_{1} C_{6} \cos \beta_{1}^{\prime} \xi-m_{2} C_{7} \sin \beta_{2} \xi+m_{2} C_{8} \cos \beta_{2} \xi .
\end{gathered}
$$

To obtain the values of the eight constants $C_{1}$ to $C_{8}$, the four boundary conditions (two for each end) and the four compatibility conditions at the cracked section must be applied, leading to a linear algebraic homogenous system

$$
M_{i k} C_{k}=0 \quad(i, k=1, \ldots, 8) .
$$

For non-trivial solutions of $C_{i}$, the determinant of the coefficients' matrix must be zero:

$$
\operatorname{det}\left(M_{i k}\right)=0 .
$$

The roots of this equation give the natural frequencies of the bending vibrations of the cracked beam. Substituting natural frequencies into Eq. (32), the corresponding mode shapes $\left(V_{1}(\xi), \Psi_{1}(\xi), V_{2}(\xi), \Psi_{2}(\xi)\right)$ are obtained.

\section{First-order perturbative solution}

The perturbation method can be applied to solve Eqs. (13) (16). The perturbative solution is obtained by extending the method originally proposed by Morassi [26] for the cracked Euler Bernoulli beam, with the assumption that the solutions for the cracked and the uncracked beams are slightly different. Since two springs have been considered connecting the two segments 
of the beam, the solutions can be expanded in Taylor series with respect to small parameters, $\varepsilon_{R}$ and $\varepsilon_{T}$, related to the flexibilities of the rotational and extensional spring, respectively. Depending on the shape of the cross-section and on the shape of the crack, the order of smallness of both flexibilities could be different. Then, for example, the solution for the vertical displacement of the first segment of the beam should be written as

$$
V_{1}(\xi)=V(\xi)+\varepsilon_{T} Y_{1 T}(\xi)+\varepsilon_{R} Y_{1 R}(\xi)+O\left(\varepsilon_{T}^{2}, \varepsilon_{R}^{2}\right)
$$

However, for the special case of the rectangular cross-section, the two flexibilities are in the same order on $\alpha\left(q(\alpha)=O\left(\alpha^{2}\right)\right.$ and $\Theta(\alpha)=O\left(\alpha^{2}\right)$, see Eqs. (11) and (12)) and, therefore, it is reasonable to expand the solution with respect to a single parameter.

Accordingly, the following expansions are introduced:

$$
\begin{gathered}
V_{1}(\xi)=V(\xi)+\varepsilon Y_{1}(\xi)+O\left(\varepsilon^{2}\right), \\
\Psi_{1}(\xi)=\Psi(\xi)+\varepsilon \varphi_{1}(\xi)+O\left(\varepsilon^{2}\right), \\
V_{2}(\xi)=V(\xi)+\varepsilon Y_{2}(\xi)+O\left(\varepsilon^{2}\right), \\
\Psi_{2}(\xi)=\Psi(\xi)+\varepsilon \varphi_{2}(\xi)+O\left(\varepsilon^{2}\right), \\
\Lambda^{2}=\lambda^{2}+\varepsilon \mu^{2}+O\left(\varepsilon^{2}\right),
\end{gathered}
$$

$\varepsilon$ being a small parameter of the same order as the flexibility of the linear springs representing the crack.

Substituting the new variables into Eqs. (13) (16) and retaining the terms of the first order, the following equations for the new variables $Y_{1}(\xi), \varphi_{1}(\xi), Y_{2}(\xi)$ and $\varphi_{2}(\xi)$ are obtained

$$
\begin{gathered}
Y_{1}^{\prime \prime}(\xi)+\lambda^{2} s^{2} Y_{1}(\xi)-\varphi_{1}^{\prime}(\xi)=-\mu^{2} s^{2} V(\xi), \quad 0<\xi<e, \\
s^{2} \varphi_{1}^{\prime \prime}(x)-\left(1-\lambda^{2} r^{2} s^{2} \varphi_{1}(\xi)\right)+Y_{1}^{\prime}(\xi)=-\mu^{2} r^{2} s^{2} \Psi(\xi), \quad 0<\xi<e, \\
Y_{2}^{\prime \prime}(\xi)+\lambda^{2} s^{2} Y_{2}(\xi)-\varphi_{2}^{\prime}(\xi)=-\mu^{2} s^{2} V(\xi), \quad e<\xi<1, \\
s^{2} \varphi_{2}^{\prime \prime}(x)-\left(1-\lambda^{2} r^{2} s^{2} \varphi_{2}(\xi)\right)+Y_{2}^{\prime}(\xi)=-\mu^{2} r^{2} s^{2} \Psi(\xi), \quad e<\xi<1 .
\end{gathered}
$$

To solve these equations, the corresponding boundary and compatibility conditions must be applied. The boundary conditions are as follows:

Simply supported end

$$
Y_{i}=\varphi_{i}^{\prime}=0
$$

Fixed end

$$
Y_{i}=\varphi_{i}=0
$$

Free end

$$
\Psi_{i}^{\prime}=0, \quad Y_{i}^{\prime}-\varphi_{i}=0 .
$$

In the above equations $i=1$ for the left end and $i=2$ for the right one. 
The compatibility conditions at the cracked section $(\xi=e)$ are as follows:

Jump in the vertical displacement:

$$
Y_{2}(e)-Y_{1}(e)=\frac{1}{\varepsilon} \frac{W}{L} \frac{r^{2}}{s^{2}} q(\alpha)\left(V_{2}^{\prime}(e)-\Psi_{2}(e)\right) .
$$

Jump in the rotation due to bending:

$$
\varphi_{2}(e)-\varphi_{1}(e)=\frac{1}{\varepsilon} \frac{W}{L} \Theta(\alpha) \Psi_{2}^{\prime}(e) .
$$

Continuity of the bending moment:

$$
\varphi_{2}^{\prime}(e)=\varphi_{1}^{\prime}(e)
$$

Continuity of the shear force:

$$
Y_{2}^{\prime}(e)-\varphi_{2}(e)=Y_{1}^{\prime}(e)-\varphi_{1}(e) .
$$

Multiplying Eqs. (40) and (42) by $V(\xi)$ and Eqs. (41) and (43) by $\Psi(\xi)$ and integrating over the whole length of the beam, the following equations can be written:

$$
\begin{aligned}
& \int_{0}^{e}\left[Y_{1}^{\prime \prime}(\xi)+\lambda^{2} s^{2} Y_{1}(\xi)-\varphi_{1}^{\prime}(\xi)\right] V(\xi) \mathrm{d} \xi \\
& +\int_{0}^{e}\left[s^{2} \varphi_{1}^{\prime \prime}(x)-\left(1-\lambda^{2} r^{2} s^{2} \varphi_{1}(\xi)\right) Y_{1}^{\prime}(\xi)\right] \Psi(\xi) \mathrm{d} \xi \\
& +\int_{e}^{1}\left[Y_{2}^{\prime \prime}(\xi)+\lambda^{2} s^{2} Y_{2}(\xi)-\varphi_{2}^{\prime}(\xi)\right] V(\xi) \mathrm{d} \xi \\
& +\int_{e}^{1}\left[s^{2} \varphi_{2}^{\prime \prime}(x)-\left(1-\lambda^{2} r^{2} s^{2} \varphi_{2}(\xi)\right)+Y_{2}^{\prime}(\xi)\right] \Psi(\xi) \mathrm{d} \xi \\
& \quad=-\mu^{2} \int_{0}^{1}\left[s^{2} V(\xi)^{2}+r^{2} s^{2} \Psi(\xi)^{2}\right] \mathrm{d} \xi .
\end{aligned}
$$

After two integrations by parts Eq. (51) becomes

$$
H_{1}+H_{2}+H_{3}+H_{4}=-\mu^{2} H_{5},
$$

where $H_{1}, H_{2}, H_{3}, H_{4}$ and $H_{5}$ have the following expressions:

$$
\begin{aligned}
H_{1}= & \int_{0}^{1}\left[\left(V^{\prime \prime}(\xi) \lambda^{2} s^{2} V(\xi)-\Psi^{\prime}(\xi)\right) f(\xi)\right. \\
& \left.+\left(s^{2} \Psi^{\prime \prime}(x)-\left(1-\lambda^{2} r^{2} s^{2} \Psi(\xi)\right)+V^{\prime}(\xi)\right) g(\xi)\right] \mathrm{d} \xi,
\end{aligned}
$$

where

$$
\begin{aligned}
f(\xi)= & \left\{\begin{array}{ll}
Y_{1}(\xi), & 0<\xi<e, \\
Y_{2}(\xi), & e<\xi<1,
\end{array} \quad g(\xi)= \begin{cases}\varphi_{1}(\xi), & 0<\xi<e, \\
\varphi_{2}(\xi), & e<\xi<1,\end{cases} \right. \\
H_{2}= & -V(0)\left(Y_{1}^{\prime}(0)-\varphi_{1}(0)\right)+Y_{1}(0)\left(V^{\prime}(0)-\Psi(0)\right) \\
& +s^{2}\left(\Psi^{\prime}(0) \varphi_{1}(0)-\Psi(0) \varphi_{1}^{\prime}(0)\right),
\end{aligned}
$$




$$
\begin{gathered}
H_{3}=V(1)\left(Y_{2}^{\prime}(1)-\varphi_{2}(1)\right)+Y_{2}(1)\left(V^{\prime}(1)-\Psi(1)\right) \\
+s^{2}\left(\Psi(1) \varphi_{2}^{\prime}(1)-\Psi^{\prime}(1) \varphi_{2}(1)\right), \\
H_{4}=\left(Y_{2}(e)-Y_{1}(e)\right)\left(V^{\prime}(e)-\Psi(e)\right)+s^{2} \Psi^{\prime}(e)\left(\varphi_{2}(e)-\varphi_{1}(e)\right) \\
-s^{2} \Psi(e)\left(\varphi_{2}^{\prime}(e)-\varphi_{1}^{\prime}(e)\right)+V(e)\left(\left(Y_{1}^{\prime}(e)-\varphi_{1}(e)\right)-\left(Y_{2}^{\prime}(e)-\varphi_{2}(e)\right)\right), \\
H_{5}=\int_{0}^{1}\left[s^{2} V(\xi)^{2}+r^{2} s^{2} \Psi(\xi)^{2}\right] \mathrm{d} \xi .
\end{gathered}
$$

Since $V(\xi)$ and $\Psi(\xi)$ are the solutions of Eq. (4) and (5), it follows that $H_{1}=0$ and taking into account the boundary conditions and the compatibility conditions, $H_{2}=H_{3}=0$ and $H_{4}$ is simplified to

$$
H_{4}=\frac{1}{\varepsilon} \frac{W}{L} \frac{r^{2}}{s^{2}} q(\alpha)\left(V^{\prime}(e)-\Psi(e)\right)^{2}+\frac{1}{\varepsilon} \frac{W}{L} \Theta(\alpha)\left(\Psi^{\prime}(e)\right)^{2} .
$$

Therefore, from Eqs. (39), (52), (58) and (59), an expression for $\Lambda^{2}$ can finally be obtained as

$$
\Lambda^{2}=\lambda^{2}-\frac{W}{L} \frac{\left(r^{2} / s^{2}\right) q(\alpha)\left(V^{\prime}(e)-\Psi(e)\right)^{2}+s^{2} \Theta(\alpha)\left(\Psi^{\prime}(e)\right)^{2}}{s^{2} \int_{0}^{1}\left(V(\xi)^{2}+r^{2} \Psi(\xi)^{2}\right) \mathrm{d} \xi} .
$$

This method provides expressions for the frequencies of bending vibrations of Timoshenko cracked beams with simple boundary conditions from the well-known natural frequencies $\left(\lambda^{2}\right)$ and normal modes of vibrations $(V(\xi)$ and $\Psi(\xi))$ of the uncracked beam.

\section{Application to a simply supported cracked beam}

In this section, the methods explained above, valid for any kind of end support, are applied to a simply supported cracked beam. The condition given by Eq. (26) is always satisfied for the cases considered in the present study. The boundary conditions for the simply supported beam are: $V(0)=\Psi^{\prime}(0)=0$ and $V(1)=\Psi^{\prime}(1)=0$. Substituting these boundary conditions and the compatibility conditions expressed by Eqs. (18) (21), in Eqs. (22) (25), the characteristic equation can be obtained (see appendix). Thus, solving this equation, the frequency parameter for the cracked beam, $\Lambda$, is calculated.

Alternatively, a first-order perturbative solution can be obtained. Following the work of Young [36], the mode shapes for a uniform simply supported uncracked beam can be written as

$$
\begin{gathered}
V(\xi)=D \sin n \pi \xi, \\
\Psi(\xi)=c D \cos n \pi \xi,
\end{gathered}
$$

where $D$ is an arbitrary constant and $n$ is the mode number. For this case, the frequency parameter $\lambda^{2}$ becomes

$$
\lambda^{2}=\frac{1+(n \pi)^{2}\left(r^{2}+s^{2}\right)-\sqrt{\left(1+(n \pi)^{2}\left(r^{2}+s^{2}\right)\right)^{2}-4(n \pi)^{4} r^{2} s^{2}}}{2 r^{2} s^{2}}
$$


and the constant $c$ in Eq. (62) is given by

$$
c=n \pi-\frac{s^{2}}{n \pi} \lambda^{2}
$$

Then, substituting Eqs. (61) (63) into Eq. (60), the following expression for $\Lambda^{2}$ is obtained:

$$
\Lambda^{2}=\lambda^{2}-2 n^{2} \pi^{2} \frac{W}{L} \frac{\left(r^{2} / s^{2}\right) q(\alpha)(1-(c / n \pi))^{2} \cos ^{2}(n \pi e)+c^{2} \Theta(\alpha) s^{2} \sin ^{2}(n \pi e)}{s^{2}\left(1+c^{2} r^{2}\right)} .
$$

For this particular case, due to the simplicity of expressions for the natural frequencies and normal modes of the uncracked beam, the perturbation method provides a closed-form expression for the natural frequencies of a cracked Timoshenko beam, that can be applied for small damages. For cracked beams with other boundary conditions, the application of Eq. (60) requires the eigenfunctions of the intact beam obtained from a $4 \times 4$ characteristic matrix and, for these cases, the perturbation method becomes less attractive.

Natural frequencies of a beam of length $L=80 \mathrm{~mm}$, width, $W=20 \mathrm{~mm}$ and thickness, $B=$ $10 \mathrm{~mm}$ with a crack in two different positions, $L^{*}=40 \mathrm{~mm}(e=0.5)$, and $L^{*}=20 \mathrm{~mm}(e=0.25)$, have been calculated using both the aforementioned methods. The following material properties have been used: $E=72 \mathrm{GPa}, G=27 \mathrm{GPa}$ and $\rho=2800 \mathrm{~kg} / \mathrm{m}^{3}$. The parameter $\kappa$ for rectangular sections is taken as $\frac{5}{6}$. Fig.2 shows the variation of natural frequency with the crack depth of a beam with a crack located at its middle section $(e=0.5)$ for the first five vibration modes. The natural frequency has been dimensionless corresponding to an uncracked Euler Bernoulli beam,

$$
\omega_{n}^{E B}=\left(\frac{n \pi}{L}\right)^{2} \sqrt{\frac{E I}{\rho A}} .
$$

The same information is shown in Fig. 3 corresponding to the case $e=0.25$. As can be seen, for dimensionless crack length $\alpha<0.3$ (shallow cracks), very small differences (less than 10\%) have been found between the values of natural frequencies predicted by the perturbation method and corresponding to the direct solution. This difference increases for higher crack lengths because the flexibility of the springs representing the crack is not small and in these cases the perturbation theory cannot be properly applied.

\section{Conclusions}

Direct and perturbative solutions for the natural frequencies for bending vibrations of cracked Timoshenko beams are provided. The beam is modelled as two segments connected by two massless springs (one extensional and another one rotational). The differential equations for the free bending vibrations are established and solved individually for each segment with the corresponding boundary conditions and the appropriated compatibility conditions at the cracked section, including discontinuities in the rotation due to bending as well as in the transverse deflection. The problem is also solved by the perturbation method, and the results obtained by both procedures are compared for the case of a simply supported cracked beam. 

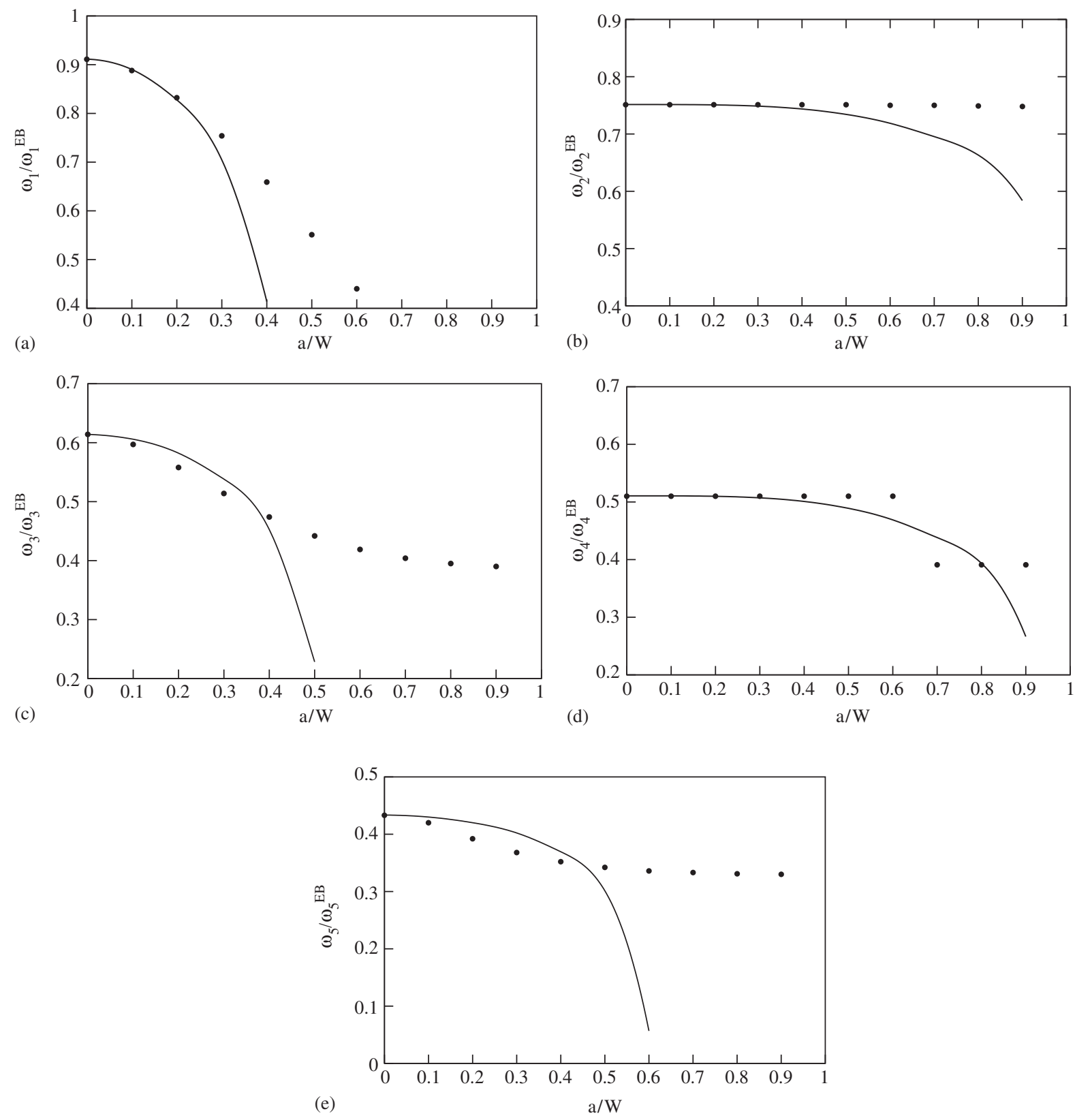

Fig. 2. Variation of natural frequencies with crack ratio of a simply supported cracked beam $(e-0.50)$. $\bullet$, Direct solution, -, perturbative solution: (a) First frequency, (b) second frequency, (c) third frequency, (d) fourth frequency and (e) fifth frequency.

The perturbation method provides simple expressions for the natural frequencies of bending vibrations of Timoshenko beams (for the case of a simply supported beam, a closed-form expression is presented). For shallow cracks $(\alpha<0.3)$, the results obtained by this method are in 

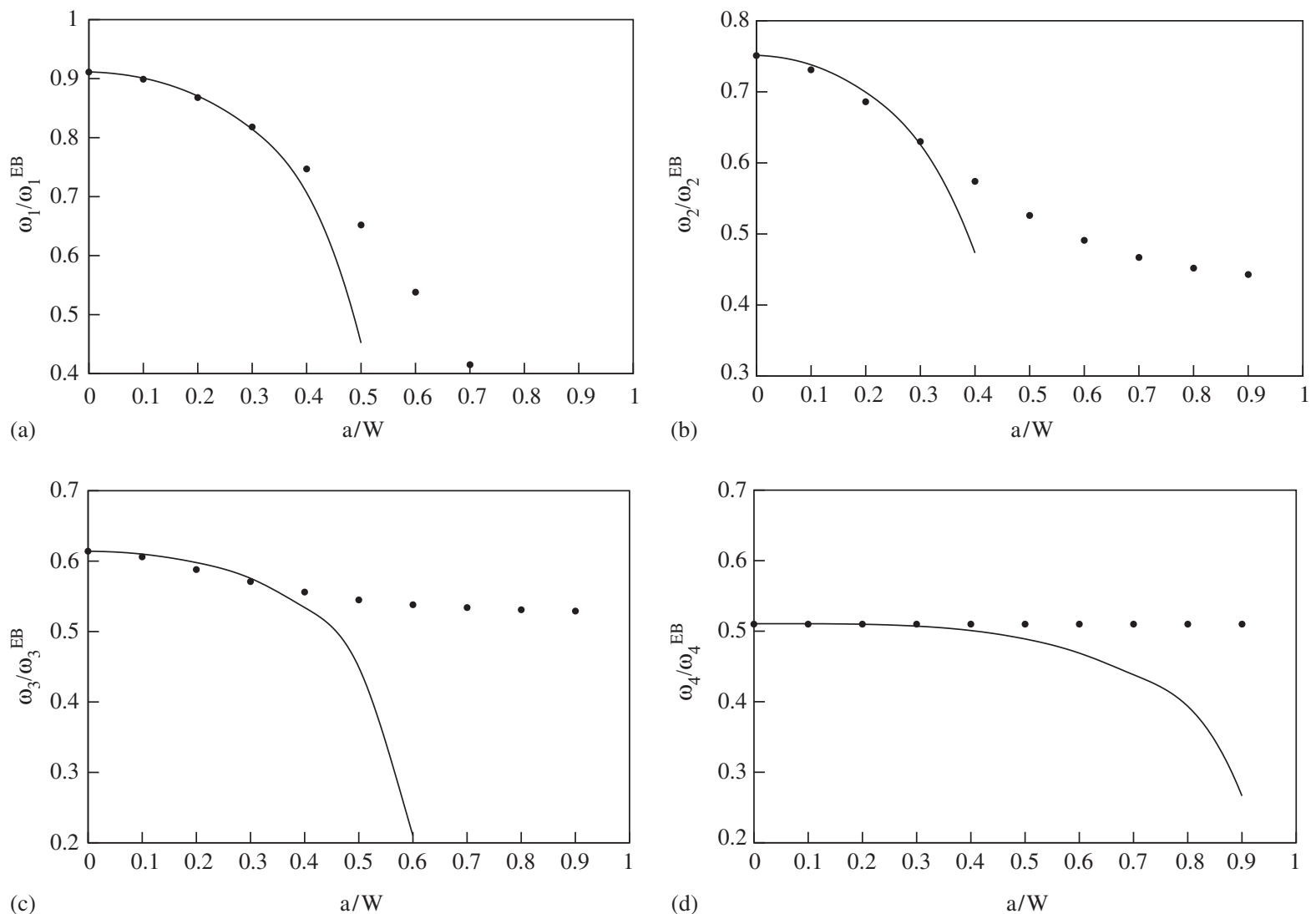

\section{(c)}

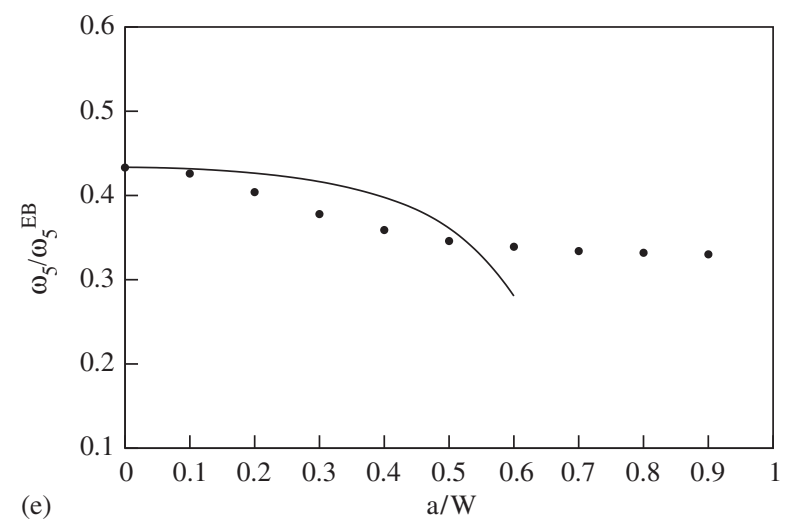

Fig. 3. Variation of natural frequencies with crack ratio of a simply supported cracked beam $(e \quad 0.25)$. $\bullet$, Direct solution, -, perturbative solution: (a) First frequency, (b) second frequency, (c) third frequency, (d) fourth frequency and (e) fifth frequency.

agreement with those derived from the direct solution, but with less computational effort. Therefore, it can be used to simplify complex crack detection algorithms, like based on optimization techniques. 


\section{Appendix A}

The characteristic equation to obtain the natural frequencies for the simply supported cracked beam is

\begin{tabular}{|c|c|c|c|c|c|c|c|}
\hline 1 & 0 & 1 & 0 & 0 & 0 & 0 & 0 \\
\hline$m_{1} \beta_{1}$ & 0 & $-m_{2} \beta_{2}$ & 0 & 0 & 0 & 0 & 0 \\
\hline$-\cosh \left(\mathrm{e} \beta_{1}\right)$ & $-\sinh \left(e \beta_{1}\right)$ & $-\cos \left(\mathrm{e} \beta_{2}\right)$ & $-\sin \left(e \beta_{2}\right)$ & $\begin{array}{c}\left(m_{1}-\beta_{1}\right) \mathrm{q} \sinh \left(\mathrm{e} \beta_{1}\right)+ \\
+\cosh \left(\mathrm{e} \beta_{1}\right)\end{array}$ & $\begin{array}{c}\left(m_{1}-\beta_{1}\right) \mathrm{q} \cosh \left(\mathrm{e} \beta_{1}\right)+ \\
\sinh \left(\mathrm{e} \beta_{1}\right)\end{array}$ & $\begin{array}{c}-\left(m_{2}-\beta_{2}\right) \mathrm{q} \sin \left(\mathrm{e} \beta_{2}\right)+ \\
+\cos \left(\mathrm{e} \beta_{2}\right)\end{array}$ & $\begin{array}{c}\left(m_{2}-\beta_{2}\right) \mathrm{q} \cos \left(\mathrm{e} \beta_{2}\right)+ \\
+\sin \left(\mathrm{e} \beta_{2}\right)\end{array}$ \\
\hline$-m_{1} \sinh \left(\mathrm{e} \beta_{1}\right)$ & $-m_{1} \cosh \left(\mathrm{e} \beta_{1}\right)$ & $m_{2} \sin \left(\mathrm{e} \beta_{2}\right)$ & $-m_{2} \cos \left(\mathrm{e} \beta_{2}\right)$ & $\begin{array}{c}m_{1}\left(-\beta_{1} \Theta \cosh \left(\mathrm{e} \beta_{1}\right)+\right. \\
\left.+\sinh \left(\mathrm{e} \beta_{1}\right)\right)\end{array}$ & $\begin{array}{c}m_{1}\left(-\beta_{1} \Theta \sinh \left(\mathrm{e} \beta_{1}\right)+\right. \\
\left.+\cosh \left(\mathrm{e} \beta_{1}\right)\right)\end{array}$ & $\begin{array}{c}m_{2}\left(\beta_{2} \Theta \cos \left(\mathrm{e} \beta_{2}\right)-\right. \\
\left.-\sin \left(\mathrm{e} \beta_{2}\right)\right)\end{array}$ & $\begin{array}{c}m_{2}\left(\beta_{2} \Theta \sin \left(\mathrm{e} \beta_{2}\right)+\right. \\
\left.+\cos \left(\mathrm{e} \beta_{2}\right)\right)\end{array}$ \\
\hline$m_{1} \beta_{1} \cosh \left(\mathrm{e} \beta_{1}\right)$ & $m_{1} \beta_{1} \sinh \left(\mathrm{e} \beta_{1}\right)$ & $-m_{2} \beta_{2} \cos \left(e \beta_{2}\right)$ & $-m_{2} \beta_{2} \sin \left(e \beta_{2}\right)$ & $-m_{1} \beta_{1} \cosh \left(\mathrm{e} \beta_{1}\right)$ & $-m_{1} \beta_{1} \sinh \left(\mathrm{e} \beta_{1}\right)$ & $m_{2} \beta_{2} \cos \left(e \beta_{2}\right)$ & $m_{2} \beta_{2} \sin \left(e \beta_{2}\right)$ \\
\hline$\left(m_{1}-\beta_{1}\right) \sinh \left(\mathrm{e} \beta_{1}\right)$ & $\left(m_{1}-\beta_{1}\right) \cosh \left(\mathrm{e} \beta_{1}\right)$ & $-\left(m_{2}-\beta_{2}\right) \sin \left(\mathrm{e} \beta_{2}\right)$ & $\left(m_{2}-\beta_{2}\right) \cos \left(\mathrm{e} \beta_{2}\right)$ & $-\left(m_{1}-\beta_{1}\right) \sinh \left(\mathrm{e} \beta_{1}\right)$ & $-\left(m_{1}-\beta_{1}\right) \cosh \left(\mathrm{e} \beta_{1}\right)$ & $\left(m_{2}-\beta_{2}\right) \sin \left(\mathrm{e} \beta_{2}\right)$ & $-\left(m_{2}-\beta_{2}\right) \cos \left(\mathrm{e} \beta_{2}\right)$ \\
\hline 0 & 0 & 0 & 0 & $\cosh \left(\beta_{1}\right)$ & $\sinh \left(\beta_{1}\right)$ & $\cos \left(\beta_{2}\right)$ & $\sin \left(\beta_{2}\right)$ \\
\hline 0 & 0 & 0 & 0 & $m_{1} \beta_{1} \cosh \left(\beta_{1}\right)$ & $m_{1} \beta_{1} \sinh \left(\beta_{1}\right)$ & $-m_{2} \beta_{2} \cos \left(\beta_{2}\right)$ & $-m_{2} \beta_{2} \sin \left(\beta_{2}\right)$ \\
\hline
\end{tabular}

\section{References}

[1] P. Cawley, R.D. Adams, The locations of defects in structures from measurements of natural frequencies, Journal of Strain Analysis 14 (1979) 4957.

[2] A.D. Dimarogonas, C.A. Papadopoulos, Vibrations of cracked shafts in bending, Journal of Sound and Vibration $91(1983) 583593$.

[3] P. Gudmundson, Eigenfrequency changes of structures due to cracks, notches or other geometrical changes, Journal of the Mechanics and Physics of Solids 30 (1982) 339353.

[4] P.F. Rizos, N. Aspragathos, A.D. Dimarogonas, Identification of crack location and magnitude in a cantilever bema from the vibration modes, Journal of Sound and Vibration 138 (1990) 381388.

[5] R.Y. Liang, F.K. Choy, J. Hu, Detection of cracks in beam structures using measurements of natural frequencies, Journal of the Franklin Institute 328 (1991) 505518.

[6] Y. Narkis, Identification of crack location in vibrating simply supported beams, Journal of Sound and Vibration 172 (1994) 549558.

[7] B.P. Nandwana, S.K. Maiti, Detection of location and size of a crack in stepped cantilever beam based on measurement of natural frequencies, Journal of Sound and Vibration 203 (1997) 435446.

[8] M.W. Suh, J.M. Yu, J.H. Lee, Crack identification using classical optimization technique, Key Engineering Materials 183187 (2000) 6166.

[9] A. Morassi, Identification of a crack in a rod based on changes in a pair of natural frequencies, Journal of Sound and Vibration 242 (2001) 577596.

[10] S.P. Lele, S.K. Maiti, Modeling of transverse vibration of short beams for crack detection and measurement of crack extension, Journal of Sound and Vibration 257 (2002) 559583.

[11] M.B. Shim, M.W. Suh, A study on multiobjective optimization technique for inverse and crack identification problems, Inverse Problems in Engineering 10 (2002) 441465.

[12] M.B. Shim, M.W. Suh, Crack identification using evolutionary algorithms in parallel computing environment, Journal of Sound and Vibration 262 (2003) 141160.

[13] Ch.Ch. Chang, L.W. Chen, Vibration damage detection of a Timoshenko beam by spatial wavelet based approach, Applied Acoustics 64 (2003) 12171240.

[14] H.P. Lin, Direct and inverse methods on free vibration analysis of simply supported beams with a crack, Engineering Structures 26 (2004) 427436.

[15] S. Christides, A.D.S. Barr, One dimensional theory of cracked Bernoulli Euler beams, International Journal of the Mechanical Sciences 26 (1984) 639648. 
[16] M. Shen, C. Pierre, Natural modes of Bernoulli Euler beams with symmetric cracks, Journal of Sound and Vibration 138 (1990) 115134.

[17] M. Shen, C. Pierre, Free vibrations of beams with a single edge crack, Journal of Sound and Vibration 170 (1994) 237259.

[18] T.G. Chondros, A.D. Dimarogonas, J. Yao, A continuous cracked beam vibration theory, Journal of Sound and Vibration 215 (1998) 1734.

[19] R.D. Adams, P. Cawley, C.J. Pye, B.J. Stone, A vibration technique for non destructive assessing the integrity of structures, Journal of Mechanical Engineering Science 20 (1978) 93100.

[20] F.D. Ju, M. Akgun, T.L. Paez, E.T. Wong, Diagnosis of fracture damage in simple structures, Bureau of Engineering Research, Report No. CE 62(82) AFOSR 993 1, University of New Mexico, Alburquerque, NM, 1982.

[21] H.P. Lee, T.Y. Ng, Natural frequencies and modes for the flexural vibration of a cracked beam, Applied Acoustics 42 (1994) 151163.

[22] G. Bamnios, A. Trochides, Dynamic behavior of a cracked cantilever beam, Applied Acoustics 45 (1995) 97112.

[23] M. Boltezar, B. Stancar, A. Kuhelj, Identification of transverse crack location in flexural vibrations of free free beams, Journal of Sound and Vibration 211 (1998) 729734.

[24] J. Fernandez Saez, L. Rubio, C. Navarro, Approximate calculation of the fundamental frequency for bending vibrations of cracked beams, Journal of Sound and Vibration 225 (1999) 345352.

[25] J. Fernandez Saez, C. Navarro, Fundamental frequency of cracked beams: an analytical approach, Journal of Sound and Vibration 256 (2002) 1731.

[26] A. Morassi, Crack induced changes in eigenfrequencies of beam structures, Journal of Engineering Mechanics 119 (1993) 17981803.

[27] W.M. Hasan, Crack detection from the variation of eigenfrequencies of a beam on elastic foundation, Engineering Fracture Mechanics 52 (1995) 409421.

[28] S.H.S. Carneiro, D.J. Inman, Continuous model for the transverse vibration of cracked Timoshenko beams, Journal of Vibration and Acoustics 124 (2002) 310320.

[29] M. Krawczuk, M. Palacz, W. Ostachowicz, The dynamic analysis of cracked Timoshenko beams by spectral element method, Journal of Sound and Vibration 264 (2003) 11391153.

[30] H. Okamura, K. Watanabe, T. Takano, Applications of compliance concept in fracture mechanics, in: J.G. Kaufman (Ed.), Progress in Flaw Growth and Fracture Toughness Testing, vol. 536, American Society for Testing and Materials, Philadelphia, 1973, pp. 423438.

[31] T. Tharp, A finite element for edge cracked beam columns, International Journal for Numerical Methods in Engineering 24 (1987) 19411950.

[32] S. Timoshenko, D. Young, W. Weaver, Vibration Problems in Engineering, Wiley, New York, 1967.

[33] T. Huang, The effect of rotary inertia and of shear deformation on the frequency and normal mode equations of uniform beams with simple end conditions, Journal of Applied Mechanics 53 (1961) 579584.

[34] A. Valiente, M. Elices, F. Ustariz, Determinacion de esfuerzos y movimientos en estructuras lineales con secciones fisuradas, Anales de Mecanica de la Fractura 7 (1990) 272277 (in Spanish).

[35] H. Tada, P. Paris, G. Irwin, The Stress Analysis of Cracks Handbook, second ed., Paris Productions, St. Louis, 1985.

[36] D. Young, Continuous systems, in: W. Flugge (Ed.), Handbook of Engineering Mechanics, McGraw Hill, New York, 1962. 